\title{
The pulmonary artery in patients with Marfan syndrome: a cross-sectional study
}

\author{
Rigmor Lundby, MD 1,2, Svend Rand-Hendriksen, MD, PhD 1,3, John K. Hald, MD, PhD², \\ Are Hugo Pripp, $\mathrm{PhD}^{4}$ and Hans-Jørgen Smith, MD, $\mathrm{PhD}^{1,2}$
}

Purpose: The objectives of this study were to establish the prevalence of pulmonary artery dilatation in Marfan syndrome using modern radiological methods and to correlate the diameter of the vessel with aortic disease.

Methods: Magnetic resonance or computed tomography imaging of the pulmonary artery and aorta was performed in 87 patients with proven Marfan syndrome. Diameters of the root and trunk of the pulmonary artery and of the aortic root were measured perpendicular to the long axes of the vessels. Pulmonary artery diameters were measured on axial images, and aortic diameters were assessed on oblique sagittal images.

Results: As compared with normal values in the literature, 47 of the 87 patients (54\%) had widening of the trunk of the pulmonary artery $(\geq 30 \mathrm{~mm})$. Of these $47,15 \%$ had no sign of disease of the ascending

\section{INTRODUCTION}

Marfan syndrome (MFS, OMIM \#154700) is an autosomal dominant genetic, connective tissue disorder characterized by manifestations in many organ systems, as described in the Ghent criteria, 1996 ("Ghent 1 criteria"). ${ }^{1}$ The diagnosis is based on findings in the skeletal system, eyes, cardiovascular and pulmonary systems, skin and integument, dura, family history, and mutations in the gene encoding fibrillin1 (FBN1). ${ }^{1,2}$ A major and frequently present finding in MFS is dilatation of the ascending aorta and/or the aortic root, and this is also the major cardiovascular criterion in the Ghent 1 nosology.

Many articles describe dilatation of the pulmonary artery as a sign of MFS, ${ }^{1,3-7}$ but documentation is sparse regarding the proportion of patients with dilated pulmonary artery in the MFS population as assessed using modern radiological methods. Although dilatation of the aortic root and of the pulmonary artery are often observed to be simultaneously present, ${ }^{3}$ it has been suggested that pulmonary artery dilatation may be a sign of cardiovascular involvement in patients with MFS even in the absence of aortic disease. ${ }^{3}$

Pulmonary artery dilatation is regularly seen in patients with pulmonary artery hypertension, and is therefore considered to be a sign of pulmonary hypertension. However, in patients with MFS, dilatation of the pulmonary artery does not seem to be related to high pressure, and it seldom results in symptoms. ${ }^{8}$ aorta. The mean (SD) ratio between the diameters of the root and trunk of the pulmonary artery was 1.18 (0.155). Multivariate analysis showed that surgery of the ascending aorta and high body surface area were associated with dilatation of the trunk of the pulmonary artery.

Conclusions: Pulmonary artery dilatation is present in a high proportion of patients with Marfan syndrome as assessed using cutoff values based on measurements in the normal population. Severe disease of the ascending aorta correlates significantly with pulmonary artery trunk dilatation in patients with Marfan syndrome.

Genet Med 2012:14(11):922-927

Key Words: aorta; connective tissue disease; Marfan syndrome; MR imaging; pulmonary artery

According to the 1996 Ghent nosology, "dilatation of the main pulmonary artery, in the absence of valvular or peripheral pulmonary stenosis or any other obvious cause, below the age of 40 years" is considered to be a minor cardiovascular criterion of MFS. ${ }^{1}$ Because normal values for the pulmonary artery were not defined, the authors recommended that dilatation of the pulmonary artery should be assessed using nomograms for normal diameters of the ascending aorta versus body surface area (BSA), as described by Roman et al. ${ }^{1,9}$

Currently, the diagnosis of MFS is based on "the revised Ghent nosology"/"the Ghent 2 criteria." Whereas pulmonary artery dilatation was listed as a minor criterion in Ghent 1 , the Ghent 2 criteria, published in $2010,{ }^{8}$ do not list dilatation of the pulmonary artery as a sign of MFS. Lack of specificity and incomplete knowledge of threshold values for dilatation of the pulmonary artery are among the reasons for this exclusion. Further research was recommended. ${ }^{8}$

This article presents data on pulmonary artery dimensions in the study population described in detail in previous studies. ${ }^{10-16}$ All investigations in patients were carried out in the period 2003-2004. Therefore all results reported in those studies are in accordance with the Ghent 1 criteria. ${ }^{1}$ The new criteria are referred to only briefly.

In this study, the axial diameters of the root and trunk of the pulmonary artery and the oblique sagittal diameter of the aortic

${ }^{1}$ Faculty of Medicine, University of Oslo, Oslo, Norway; ${ }^{2}$ Department of Radiology and Nuclear Medicine, Oslo University Hospital, Oslo, Norway; ${ }^{3}$ TRS National Resource Centre for Rare Disorders, Sunnaas Rehabilitation Hospital, Nesoddtangen, Norway; ${ }^{4}$ Biostatistics and Epidemiology Unit, Oslo University Hospital, Oslo, Norway. Correspondence: Rigmor Lundby (rigmor.lundby@medisin.uio.no) 
root were measured using magnetic imaging (MR) imaging or computed tomography (CT) in a cohort of 87 patients who had proven MFS and no other known causes of pulmonary artery dilatation. The measurements were compared with normal values measured using MR and CT, as reported in the literature. The aims were to assess the prevalence of pulmonary artery dilatation in MFS and to correlate this with aortic disease.

\section{MATERIALS AND METHODS}

The study was approved by the regional medical ethics committee. In order to comply with the requirement for informed consent, only patients $>18$ years of age were included in the study.

None of the patients was thought to have pulmonary artery hypertension.

\section{Study population}

The participants were recruited through (i) letters sent to the 134 individuals $>18$ years of age who were registered in a national resource center as having MFS, (ii) an advertisement in the journal of the National Association of MFS, and (iii) invitations distributed in the Department of Thoracic and Cardiovascular Surgery at our hospital to patients suspected of having MFS. Of the 109 individuals who gave informed consent for participation, one died before the study started, one who was living abroad was not able to attend, and two withdrew. The remaining 105 participants were all Caucasians. All the patients were assessed for all subsets of the Ghent criteria (Ghent 1$)^{1}$ by the same group of physicians. ${ }^{10-16}$ For assessing fulfilment of the major cardiovascular criterion in the Ghent nosology, information was collected about previous surgery related to dilatation or dissection of the ascending aorta, and electrocardiographic evidence of dilatation of the ascending aorta was recorded.,13 The assessment of fulfilment of the Ghent criteria included sequencing of the entire coding region of the gene FBN1 and searching for large deletions or duplications. ${ }^{12,13,16}$

\section{MR or CT of the aorta and pulmonary artery}

This study was a substudy of the Norwegian Marfan Study. In this substudy, MR imaging of the aorta and the pulmonary artery was performed except when MR was contraindicated, in which case CT images were obtained instead.

MR imaging at $1.5 \mathrm{~T}$ (Signa LX; GE Healthcare, Milwaukee, WI) of the aorta and pulmonary artery in axial and oblique sagittal planes was performed using a standard ECG-gated T1-weighted SE sequence. Repetition time was equal to the $\mathrm{R}-\mathrm{R}$ interval, echo time was $9.32 \mathrm{~ms}$ (minimum) to $39.976 \mathrm{~ms}$ (maximum), and slice thicknesses were $7 \mathrm{~mm}$ in the axial plane and $4 \mathrm{~mm}$ in the oblique sagittal plane.

Nonenhanced axial CT images with 5-mm slice thickness were obtained on a ProSpeed SX scanner (GE Healthcare) from patients in whom MR imaging was contraindicated. As part of the assessment of all Ghent criteria, high-resolution CT of the lungs was also performed.

\section{Measurements and estimations}

All MR and CT results were subjected to consensus verification by two radiologists (H.J.S. and R.L.). Aortic dimensions were assessed perpendicular to the long axis of the vessel on oblique sagittal images at the level of the sinuses of Valsalva (aortic root). Main pulmonary artery dimensions were assessed perpendicular to the long axis of the artery on axial images at two levels: the root, and the trunk at the point of bifurcation (Figure 1).

In this substudy, fulfillment of the major cardiovascular criterion for MFS was reassessed using MR or CT measurements of all native ascending aortas. For each participant, the diameter of the aortic root and the BSA were plotted in Roman et al.s nomogram.,

This major criterion was also considered to be fulfilled in participants who had undergone surgery on the ascending aorta because of dilatation or dissection.

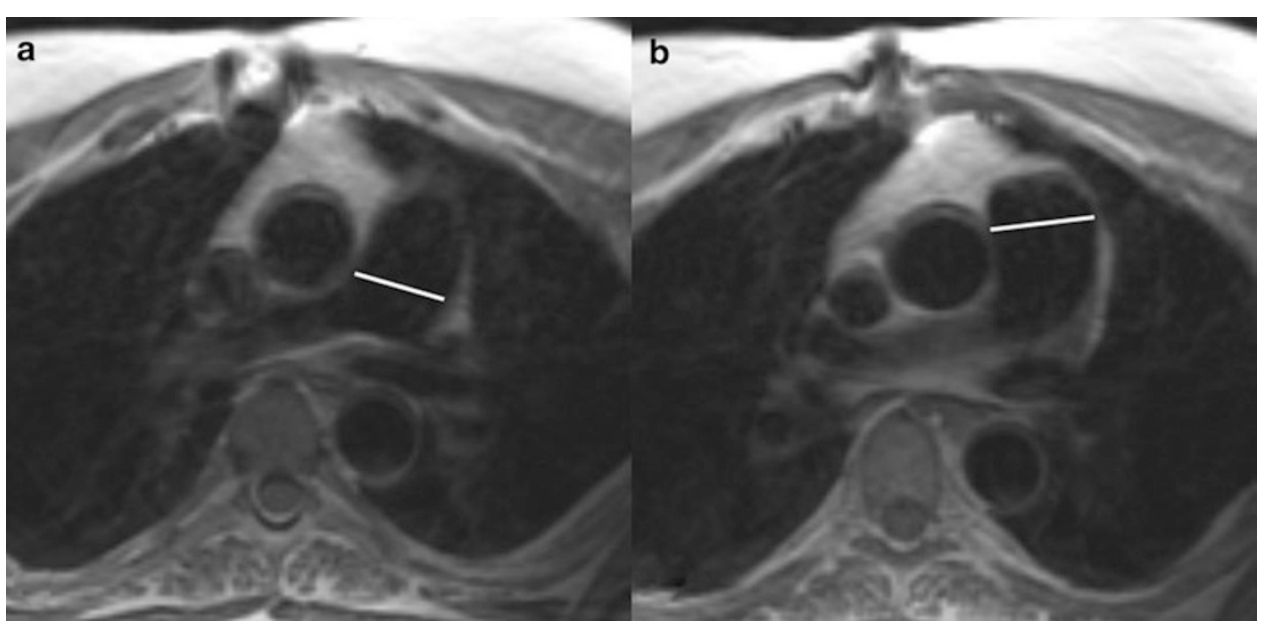

Figure 1 Axial MR images of a 63-year-old male patient with MFS. Measurements of the diameters of (a) the trunk and (b) the root of the pulmonary artery are indicated. The ascending aorta has been replaced with a graft. MFS, Marfan syndrome; MR, magnetic resonance. 
Assessment of pulmonary artery dilatation (root and trunk) as per the Ghent 1 criteria was performed using nomograms for the aortic root. ${ }^{1}$ Dilatation of the trunk of the pulmonary artery was reassessed by comparing the data with recently published normal values based on 5-mm axial CT imaging. The latter suggest that a mean diameter of $24.0 \mathrm{~mm}$ with an upper limit of $29.6 \mathrm{~mm}(24.0 \mathrm{~mm}+2 \mathrm{SD})$ can be considered to be the dimensions of the normal pulmonary artery trunk in adults. ${ }^{17}$ In our reassessment, we took measurements using electronic callipers with $1-\mathrm{mm}$ increments, and defined pulmonary trunk dilatation as being present when the diameter was $\geq 30 \mathrm{~mm}$.

\section{Statistics}

Data were expressed in terms of mean (SD) values and frequency (percentage) for continuous and categorical variables, respectively. Univariate statistical analysis of differences between groups were analyzed using independent sample $t$-test or $\chi^{2}$ test, as appropriate, and correlations were assessed with the Pearson correlation coefficient. Multivariate analysis of factors influencing pulmonary artery diameter was carried out using a multiple linear model. The statistical level of significance was 5\%. All analyses were conducted using SPSS version 18 (SPSS, Chicago, IL).

\section{RESULTS}

Of the 105 individuals in the study (56 of whom were women), 87 fulfilled the Ghent 1 criteria for MFS. These 87 persons constituted the study group in this substudy, which was part of a larger national MFS study. The mean (SD) age of the men was 35.1 (11.1) years, and of the women was 42.1 (13.5) years. The characteristics of the study population are given in Table 1.

The results related to the major criteria fulfilled and the organ systems involved have been published previously. ${ }^{13} \mathrm{~A}$ total of 73 (83.9\%) of our Ghent-positive patients had an FBN1 mutation, and $54(62.1 \%)$ had ectopia lentis. As per echocardiographic findings, none of the participants had high pressure in the pulmonary artery.

MR imaging was contraindicated in six individuals, and CT was performed instead. In five of the 81 thoracic MR examinations of the study group, image quality was inadequate for measurement of pulmonary artery dimensions; in these five cases, high-resolution CT was available for diameter measurements. In one of the 87 patients, neither MR nor CT showed adequate imaging of the root of the pulmonary artery, and therefore the diameter could not be measured.

MR and CT measurements of the pulmonary artery showed mean (SD) diameters of the root and trunk of the pulmonary artery to be 35.0 (4.6) $\mathrm{mm}$ and 29.8 (3.8) $\mathrm{mm}$, respectively. The root of the pulmonary artery was found to have a larger diameter than the trunk in most cases, the mean ( $\mathrm{SD}$, range) ratio between the two measurements being 1.18 (0.155, 0.78-1.58).

When assessed according to Ghent 1 criteria for pulmonary artery dilatation, ${ }^{1}$ which are based on nomograms of the aortic root, 7 of the 39 patients $(17.9 \%)<40$ years of age had dilatation of the root of the pulmonary artery, whereas none showed dilatation of the trunk of the vessel. When assessed by comparison with published normal values in adults, ${ }^{17} 47$ (54\%) of the 87 patients had dilatation of the trunk of the pulmonary artery, the diameter being $\geq 30 \mathrm{~mm}$.

A total of 30 (34.5\%) of the 87 persons had undergone surgery on the ascending aorta. Echocardiographic measurements in the other 57 patients showed that 16 of them had dilatation of the aortic root as per the Ghent 1 criteria (nomograms by Roman et al. $)^{9}$; however, when the aortic root diameters were reassessed using MR or CT, 40 of the 57 aortic roots were found to be dilated. On the basis of MR or CT, a total of $70(80.5 \%)$ patients fulfilled the major cardiovascular criterion for MFS. The mean (SD) aortic root diameter as measured by MR or CT in the 57 patients with native ascending aorta was $41.3(6.1) \mathrm{mm}$.

Of the 47 patients in whom the pulmonary artery trunk diameter was $\geq 30 \mathrm{~mm}$, seven had normal diameters of the aortic root, $20(42.6 \%)$ had dilatation of the native aortic root, and $20(42.6 \%)$ had undergone surgery on the ascending aorta.

There were no significant differences between genders with respect to pulmonary artery diameters, but there was a

Table 1 Characteristics of study population given as mean (SD) or number of patients (percentage)

\section{Patient characteristic}

\begin{tabular}{|c|c|}
\hline Number of patients & 87 \\
\hline Female & $56(64.4 \%)$ \\
\hline Age (years) & $39.6(13.0)$ \\
\hline Weight (kg) & $82.7(17.3)$ \\
\hline Height $(\mathrm{cm})$ & $184.2(10.6)$ \\
\hline $\mathrm{BSA}\left(\mathrm{m}^{2}\right)$ & $2.07(0.24)$ \\
\hline $\mathrm{BMI}\left(\mathrm{kg} / \mathrm{cm}^{2}\right)$ & $24.7(4.38)$ \\
\hline FBN1 mutation & $73(83.9 \%)$ \\
\hline Ectopia lentis & $54(62.1 \%)$ \\
\hline Mitral valve prolapse & $12(13.8 \%)$ \\
\hline Calcification of the mitral annulus before age of 40 & $1(1.1 \%)$ \\
\hline Patients with previous surgery on ascending aorta & $30(34.5 \%)$ \\
\hline $\begin{array}{l}\text { Diameter of aortic roots }(\mathrm{mm}) \text { without previous } \\
\text { aortic surgery, } n=56\end{array}$ & $36.6(6.9)$ \\
\hline Pulmonary artery root diameter $(\mathrm{mm}), n=86$ & $35.0(4.6)$ \\
\hline Pulmonary artery trunk diameter (mm) & $29.8(3.8)$ \\
\hline $\begin{array}{l}\text { Pulmonary artery trunk widened according to Ghent } \\
1 \text { criteria (1996) }\end{array}$ & 0 \\
\hline $\begin{array}{l}\text { Pulmonary artery root widened according to Ghent } 1 \\
\text { criteria (1996) }\end{array}$ & 7 \\
\hline $\begin{array}{l}\text { Pulmonary artery trunk widened according to cut-off } \\
30 \mathrm{~mm}\end{array}$ & $47(54 \%)$ \\
\hline $\begin{array}{l}\text { Pulmonary artery root widened according to cut-off } \\
34.8 \mathrm{~mm}, n=86\end{array}$ & $43(50 \%)$ \\
\hline
\end{tabular}

Diameters measured by MR or CT.

$\mathrm{BMI}$, body mass index; BSA, body surface area; $\mathrm{CT}$, computed tomography; MR, magnetic resonance. 
tendency toward larger values for the diameter of the pulmonary artery root in men $(P=0.063)$ (Table 2$)$. No correlation was found between dilatation of the trunk of the pulmonary artery and the presence of FBN1 mutation or of ectopia lentis ( $P=0.359$ and $P=0.418$, respectively). The diameters of the roots of the pulmonary artery were significantly correlated with age, height, weight, body mass index, and BSA, whereas the diameters of the trunks had significant positive correlation with weight and BSA only (Table 2). Both root and trunk diameters were significantly larger in patients who had undergone surgery on the ascending aorta as compared with those who had not undergone such surgery ( $P=0.041$ and $P=0.027$, respectively) (Table 2). Pulmonary artery trunk diameters were significantly larger in patients with disease of the ascending aorta (dilatation or previous surgery) than in those without $(P=0.018)$, but the pulmonary artery root diameters were not significantly larger in this patient group $(P=0.104)$.

The results of a multivariate analysis of variables that could potentially have influenced the root and trunk diameters of the pulmonary artery are shown in Table 3. For the root of the artery, age $(P=0.009)$ and BSA $(P=0.028)$ were the only parameters that correlated significantly with the diameter. Previous surgery on the ascending aorta $(P=0.005)$ or BSA $(P=0.004)$ was significantly correlated with the diameter of the trunk of the pulmonary artery.

\section{DISCUSSION}

Our study showed that, as compared with normal values in healthy adult subjects, ${ }^{17}$ more than half (54\%) of our adult patients with proven MFS had dilatation of the trunk of the pulmonary artery, some $(\sim 15 \%)$ even in the absence of dilatation of the aortic root or the ascending aorta.

The 1996 Ghent 1 criteria $^{1}$ included dilatation of the pulmonary artery as a sign (minor vascular criterion) of MFS, but in

Table 2 Pulmonary artery diameters $(\mathrm{mm})$ in relation to patient characteristics

\begin{tabular}{|c|c|c|c|c|}
\hline & \multicolumn{2}{|c|}{$\begin{array}{l}\text { Pulmonary artery } \\
\text { root, } n=86\end{array}$} & \multicolumn{2}{|c|}{$\begin{array}{l}\text { Pulmonary artery } \\
\text { trunk, } n=87\end{array}$} \\
\hline & $\begin{array}{c}\text { Mean (SD)/ } \\
\text { correlation }(r)\end{array}$ & $\begin{array}{c}P \\
\text { value }\end{array}$ & $\begin{array}{c}\text { Mean (SD)/ } \\
\text { correlation }(r)\end{array}$ & $\begin{array}{c}P \\
\text { value }\end{array}$ \\
\hline \multicolumn{5}{|l|}{ Gender } \\
\hline Male & $36.2(4.2)$ & 0.063 & $29.9(3.1)$ & 0.932 \\
\hline Female & $34.4(4.7)$ & & $29.8(4.2)$ & \\
\hline Age & $r=0.300$ & 0.005 & $r=0.182$ & 0.091 \\
\hline Height & $r=0.261$ & 0.015 & $r=0.147$ & 0.173 \\
\hline Weight & $r=0.425$ & $<0.001$ & $r=0.353$ & 0.001 \\
\hline BMI & $r=0.265$ & 0.014 & $r=0.185$ & 0.086 \\
\hline BSA & $r=0.385$ & $<0.001$ & $r=0.285$ & 0.007 \\
\hline \multicolumn{5}{|c|}{ Previous ascending aorta surgery } \\
\hline Yes, $n=30$ & $36.5(5.2)$ & 0.041 & $31.3(4.8)$ & 0.027 \\
\hline No & $\begin{array}{c}34.2(4.1) \\
n=56\end{array}$ & & $\begin{array}{c}29.1(3.0) \\
n=57\end{array}$ & \\
\hline
\end{tabular}

BMI, body mass index; BSA, body surface area. the revised criteria (Ghent 2) published in $2010,{ }^{8}$ this criterion was omitted. The reasons given were that pulmonary artery dilatation is not specific to the diagnosis of MFS, that complications of pulmonary artery disease are rarely seen, and that further research is needed regarding thresholds and the diagnostic utility of this finding. ${ }^{8}$ The uncertainty regarding threshold values for pulmonary artery dilatation is attributable partly to the vagueness of the wording of the Ghent 1 criteria: "until normal values for pulmonary artery diameter are available, dilatation can be detected provisionally by echocardiography, CT, or MR imaging, using nomograms for the aorta." ${ }^{\prime}$ Further, the Ghent 1 criteria do not specify which part of the pulmonary artery should be measured-the root or the trunk.

In our study of 87 MFS patients, we measured the root and trunk diameters of the pulmonary artery on axial 7-mm (for $\mathrm{MR}$ ) or 5-mm (for CT) slices. In the literature, most of the papers that have addressed upper normal values for the pulmonary artery have considered only the trunk of the pulmonary artery, and among those performed using axial imaging, $4,17-21$ only four included an adequate number of subjects (Table 4). The upper normal limit for the trunk diameter of the pulmonary artery, defined as mean diameter $+2 \mathrm{SD}$, was clearly larger in two of the studies ${ }^{18,19}$ than in the other two, ${ }^{17,21} 32.4$ and $33.2 \mathrm{~mm}$ vs. 29.6 and $30.1 \mathrm{~mm}$, respectively. This may be explained by the difference in slice thickness, $10 \mathrm{~mm}$ vs. $5 \mathrm{~mm}$ and $2.5 \mathrm{~mm}$. Axial slices run obliquely across the main pulmonary artery, and thicker slices are apt to lead to overestimation of diameter because of partial volume effects. The 126 subjects studied by Bozlar et al. ${ }^{17}$ were imaged using nearly the same slice thickness as the patients with MFS in our study, and the age distribution was not very dissimilar, 30.6 \pm 7.9 and $39.6 \pm 13$

Table 3 Multivariate analysis of variables that might affect pulmonary artery diameter

\begin{tabular}{lll} 
Parameter & Coefficient $(95 \%$ Cl) & $P$ value \\
\hline $\begin{array}{l}\text { A. Dependent variable: diameter of pulmonary artery root } \\
\text { Ascending aorta }\end{array}$ & \\
\hline Dilated & $0.73(-1.68 ; 3.15)$ & 0.55 \\
\hline Previous surgery & $2.00(-0.54 ; 4.54)$ & 0.12 \\
\hline Male gender & $0.77(-1.67 ; 3.22)$ & 0.53 \\
\hline Age & $0.098(0.025 ; 0.17)$ & 0.009 \\
\hline BSA & $5.38(0.60 ; 10.16)$ & 0.028 \\
\hline BMI & $0.13(-0.098 ; 0.35)$ & 0.27 \\
\hline B. Dependent variable: diameter of the pulmonary artery trunk & \\
\hline Ascending aorta & $1.68(-0.42 ; 3.77)$ & 0.12 \\
\hline Dilated & $3.18(-0.97 ; 5.39)$ & 0.005 \\
\hline Previous surgery & $-1.96(-4.08 ; 0.16)$ & 0.07 \\
\hline Male gender & $0.02(-0.04 ; 0.09)$ & 0.48 \\
\hline Age & $6.2(2.03 ; 10.36)$ & 0.004 \\
\hline BSA & $0.017(-0.18 ; 0.21)$ & 0.86 \\
\hline BMI &
\end{tabular}

$\mathrm{BMI}$, body mass index; $\mathrm{BSA}$, body surface area; $\mathrm{Cl}$, confidence interval. 
Table 4 Literature review of normal PA trunk diameters based on axial CT or MR imaging

\begin{tabular}{|c|c|c|c|c|c|}
\hline Reference & Subjects $(N)$ & Age, mean \pm SD (years) & $\begin{array}{l}\text { Modality/slice } \\
\text { thickness }\end{array}$ & $\begin{array}{l}\text { PA trunk diameter, } \\
\text { mean } \pm \text { SD }(\mathrm{mm})\end{array}$ & $\begin{array}{l}\text { PA trunk upper limit, } \\
\text { mean }+2 \text { SD (mm) }\end{array}$ \\
\hline Karazincir et al., $2008^{19}$ & 112 & $46.3 \pm 13.6$ & $\mathrm{CT} / 10 \mathrm{~mm}$ & $26.6 \pm 2.9$ & 32.4 \\
\hline Bozlar et al., $2007^{17}$ & 126 & $30.6 \pm 7.9$ & $\mathrm{CT} / 5 \mathrm{~mm}$ & $24.0 \pm 2.8$ & 29.6 \\
\hline Nollen et al., $2002^{4}$ & 15 & $28.0 \pm 4.0$ & MR/unknown & $24.0 \pm 2.0$ & 28.0 \\
\hline Choe et al., 200020 & 10 & Unknown, range $25-72$ & $\mathrm{CT} / 10 \mathrm{~mm}$ & $25.6 \pm 2.2$ & 30.0 \\
\hline
\end{tabular}

$\mathrm{CT}$, computed tomography; MR, magnetic resonance; PA, pulmonary artery.

years, respectively. Pulmonary hypertension and thoracic pathology had been excluded. We therefore consider this group of healthy adults as an adequate control group for our study. In the largest study, the so-called Framingham study, ${ }^{21}$ the 706 healthy persons studied had a mean age 6 years greater than that of our study population ( 45.6 vs. 39.6 years), and CT was performed with half the slice thickness. Cutoff values for pulmonary artery diameters were given as 90 th percentile $(29 \mathrm{~mm}$ for men and $27 \mathrm{~mm}$ for women), but when given as mean +2 $\mathrm{SD}$ for the whole population, the result $(30.1 \mathrm{~mm})$ is quite similar to that reported by Bozlar et al. $(29.6 \mathrm{~mm})$. The data from the Framingham study therefore support our choice of cutoff value for the trunk diameter of the pulmonary artery $(30 \mathrm{~mm})$.

Normal values for the root diameter of the pulmonary artery are nearly nonexistent in the literature. Nollen et al. ${ }^{4}$ measured the diameters of the roots and trunks of the pulmonary artery in 50 patients with MFS and in 15 controls, using MR imaging. However, they did not measure the root diameters perpendicular to the long axis of the vessel; instead, three separate oblique diameters in the axial plane were measured, and the anterior right diameter was used for analysis. On that basis, they suggested an upper normal value of $34.8 \mathrm{~mm}$ for the root diameter of the pulmonary artery. Using this cutoff value, 50\% (43 of 86) of the patients with MFS in our study had dilatation of the root of the pulmonary artery (Table 1). The root diameter in patients and controls has also been assessed using echocardiography and, based on receiver operating characteristic analysis, a cutoff value of $23 \mathrm{~mm}$ was suggested for distinguishing between normal individuals and those with MFS in subjects $>14$ years of age. ${ }^{3}$ This is clearly a much smaller value than the upper limit suggested by Nollen et al. ${ }^{4}(34.8 \mathrm{~mm})$ and the mean value in the patients in our study $(35.0 \mathrm{~mm})$, which probably reflects the inherent differences between the modalities used. During echocardiography, diameter measurements are usually performed from leading edge to leading edge, thereby excluding the thickness of the distant wall, whereas CT and MR measurements often include both vessel walls. Further, echocardiography is dependent on an acoustic window, and may therefore be incapable of obtaining an optimal image plane. These differences in imaging techniques are underscored by the findings in our study. Our preliminary measurements of aortic root diameters were performed using echocardiography and resulted in 16 of 57 patients being identified as having dilatation of the aortic root. When re-measured using MR or CT, the number increased to 40 of 57 .

With regard to pulmonary artery dilatation, there was a large discrepancy between the findings using nomograms for the aorta as recommended by Ghent $1^{1}$ and those using cutoff values set at $2 \mathrm{SD}$ above the mean value in healthy volunteers. This suggests that nomograms for the aorta are not suited for assessment of pulmonary artery dilatation. In the normal population, the trunk of the pulmonary artery is, on average, smaller than the ascending aorta, but the variations are large. In a recent multidetector CT study of 103 normal, healthy adults, the mean (SD) ratio of the diameters of short-axis pulmonary trunk to short-axis ascending aorta was $0.89(0.24){ }^{22}$

The roots of the aortic and pulmonary arteries have a common embryologic origin. ${ }^{23}$ Given that aortic root dilatation is typical of MFS, it has been speculated that the root of the pulmonary artery is the most likely part of the vessel to experience dilatation. ${ }^{3,4}$ There is only sparse evidence to support this in the literature. In an MR study of 50 adult patients with MFS and 15 adult controls, ${ }^{4}$ the root of the pulmonary artery was found to be wider than the trunk in both patients and controls; however, this difference was larger in the patients than in the controls. ${ }^{4}$ The pulmonary artery root being wider than the trunk is confirmed by the findings of our study, the mean (SD) diameters being 35.0 (4.6) $\mathrm{mm}$ and 29.8 (3.8) $\mathrm{mm}$, respectively.

It could be hypothesized on the basis of these findings that root dilatation of the pulmonary artery rather than trunk dilatation is more strongly correlated to disease of the ascending aorta in patients with MFS. This was not confirmed by our study. Both the root and the trunk of the pulmonary artery were significantly larger in patients who had undergone surgery on the ascending aorta than in those who had not, probably reflecting a correlation between pulmonary artery dilatation and the most severe cases of aortic disease in MFS. However, when comparing pulmonary artery diameters in patients fulfilling the major cardiovascular criterion (either surgery or dilatation of the aortic root or ascending aorta) and those not fulfilling the criterion, only the trunk diameter was found to be significantly larger in patients who fulfilled the criterion. A correlation between aortic disease and pulmonary artery trunk diameter was also suggested by the findings of our multivariate analysis. The results showed that previous surgery on the ascending aorta correlated significantly with 
dilatation of the trunk of the pulmonary artery but not with dilatation of the root.

Although a large majority of the patients with dilatation of the trunk of the pulmonary artery also had aortic disease (40 of 47 , $85.1 \%$ ), a few of these patients (7 of $47,14.9 \%$ ) had no sign of ascending aortic disease, suggesting that pulmonary artery dilatation may be an early marker of vascular disease in MFS patients.

The clinical implications of our findings are few, but important. Dilatation of the trunk of the pulmonary artery is common in patients with MFS, and does not by itself imply pulmonary hypertension. Dilatation of this vessel in a patient with MFS may suggest MFS-related vascular effects even in the absence of visible disease of the ascending aorta.

\section{Limitations}

A major limitation of our study is the lack of controls in whom exactly the same imaging techniques were used as in the patients. However, some relevant, high-quality studies in healthy adults have been published, using techniques similar to ours. We therefore believe that normal values for the diameter of the pulmonary artery measured using axial imaging are now well documented. ${ }^{17,21}$ However, reliable values for the normal root diameter are not yet available, and may be difficult to obtain given the asymmetry of this part of the vessel.

Our study was performed in the period 2002-2004, and MR techniques for nonenhanced imaging of thoracic vessels have improved since then. Multi-slice, ECG-triggered MR imaging also introduces a small variation in vessel diameter because of imaging of the vessel at different time points during the cardiac cycle.

\section{Conclusions}

Pulmonary artery dilatation is a common finding in MFS (54\%) and should be assessed using cutoff values based on pulmonary artery diameter measurements in the normal population, and not on nomograms intended for the aorta. MR or CT imaging seems to provide more reliable results for pulmonary artery dimensions than echocardiography. Severe disease of the ascending aorta correlates significantly with dilatation of the trunk of the pulmonary artery in patients with MFS, and such dilatation may occur even in the absence of visible aortic disease.

\section{DISCLOSURE}

The authors declares no conflict of interest.

\section{REFERENCES}

1. De Paepe A, Devereux RB, Dietz HC, Hennekam RC, Pyeritz RE. Revised diagnostic criteria for the Marfan syndrome. Am J Med Genet 1996;62: 417-426.

2. Pyeritz RE. The Marfan syndrome. Annu Rev Med 2000;51:481-510.
3. De Backer J, Loeys B, Devos D, Dietz H, De Sutter J, De Paepe A. A critical analysis of minor cardiovascular criteria in the diagnostic evaluation of patients with Marfan syndrome. Genet Med 2006;8:401-408.

4. Nollen GJ, van Schijndel KE, Timmermans J, et al. Pulmonary artery root dilatation in Marfan syndrome: quantitative assessment of an unknown criterion. Heart 2002;87:470-471.

5. Nollen GJ, Mulder BJ. What is new in the Marfan syndrome? Int J Cardiol 2004;97(suppl 1):103-108.

6. Ha HI, Seo JB, Lee SH, et al. Imaging of Marfan syndrome: multisystemic manifestations. Radiographics 2007;27:989-1004.

7. McKusick VA. The cardiovascular aspects of Marfan's syndrome: a heritable disorder of connective tissue. Circulation 1955;11:321-342.

8. Loeys BL, Dietz HC, Braverman AC, et al. The revised Ghent nosology for the Marfan syndrome. J Med Genet 2010;47:476-485.

9. Roman MJ, Devereux RB, Kramer-Fox R, O'Loughlin J. Two-dimensional echocardiographic aortic root dimensions in normal children and adults. Am J Cardiol 1989;64:507-512.

10. Lundby R, Rand-Hendriksen S, Hald JK, et al. Dural ectasia in Marfan syndrome: a case control study. AJNR Am J Neuroradiol 2009;30: 1534-1540.

11. Lundby R, Kirkhus E, Rand-Hendriksen S, Hald J, Pripp AH, Smith HJ. CT of the hips in the investigation of protrusio acetabuli in Marfan syndrome. A case control study. Eur Radio/ 2011;21:1485-1491.

12. Rand-Hendriksen $S$, Tjeldhorn L, Lundby R, et al. Search for correlations between FBN1 genotype and complete Ghent phenotype in 44 unrelated Norwegian patients with Marfan syndrome. Am J Med Genet A 2007;143A:1968-1977.

13. Rand-Hendriksen $S$, Lundby $R$, Tjeldhorn $L$, et al. Prevalence data on all Ghent features in a cross-sectional study of 87 adults with proven Marfan syndrome. Eur J Hum Genet 2009;17:1222-1230.

14. Rand-Hendriksen S, Johansen H, Semb SO, Geiran O, Stanghelle JK, Finset A. Health-related quality of life in Marfan syndrome: a cross-sectional study of Short Form 36 in 84 adults with a verified diagnosis. Genet Med 2010;12:517-524.

15. Rand-Hendriksen S. Marfan syndrome - a diagnostic challenge: aspects of a Norwegian cohort study. [Clinical Medicine: University of Oslo, Norway, 2010.]

16. Tjeldhorn L, Rand-Hendriksen S, Gervin K, et al. Rapid and efficient FBN1 mutation detection using automated sample preparation and direct sequencing as the primary strategy. Genet Test 2006;10: 258-264.

17. Bozlar U, Ors F, Deniz O, et al. Pulmonary artery diameters measured by multidetector-row computed tomography in healthy adults. Acta Radiol 2007:48:1086-1091

18. Edwards PD, Bull RK, Coulden R. CT measurement of main pulmonary artery diameter. Br J Radiol 1998;71:1018-1020.

19. Karazincir S, Balci A, Seyfeli E, et al. CT assessment of main pulmonary artery diameter. Diagn Interv Radiol 2008;14:72-74.

20. Choe KO, Hong YK, Kim HJ, et al. The use of high-resolution computed tomography in the evaluation of pulmonary hemodynamics in patients with congenital heart disease: in pulmonary vessels larger than $1 \mathrm{~mm}$ in diameter. Pediatr Cardiol 2000;21:202-210.

21. Truong QA, Massaro JM, Rogers IS, et al. Reference values for normal pulmonary artery dimensions by noncontrast cardiac computed tomography: the Framingham Heart Study. Circ Cardiovasc Imaging 2012;5:147-154.

22. Lin FY, Devereux RB, Roman MJ, et al. The right sided great vessels by cardiac multidetector computed tomography: normative reference values among healthy adults free of cardiopulmonary disease, hypertension, and obesity. Acad Radio/ 2009; 16:981-987.

23. Brown $C B$, Baldwin HS. Neural crest contribution to the cardiovascular system. Adv Exp Med Bio/ 2006;589:134-154. 